\title{
ON POSITIVE SOLUTIONS OF SOME SEMILINEAR ELLIPTIC EQUATIONS
}

\author{
SHIN-HWA WANG and NICHOLAS D. KAZARINOFF
}

(Received 15 February 1989; revised 19 March 1990)

Communicated by E. N. Dancer

\begin{abstract}
The existence of positive solutions of some semilinear elliptic equations of the form $-\Delta u=$ $\lambda f(u)$ is studied. The major results are a nonexistence theorem which gives a $\lambda^{*}=\lambda^{*}(f, \Omega)>0$ below which no positive solutions exist and a lower bound theorem for $u_{\max }$ for $\Omega$ a ball. As a corollary of the nonexistence theorem that describes the dependence of the number of solutions on $\lambda$, two other nonexistence theorems, and an existence theorem are also proved.
\end{abstract}

1980 Mathematics subject classification (Amer. Math. Soc.) (1985 Revision): $35 \mathrm{~J} 65,35 \mathrm{~J} 25$. Keywords and phrases: positive solution, semilinear elliptic equation, degree theory.

\section{Introduction}

We study the existence of positive solutions $u$ in $C^{2}(\Omega) \cap C(\bar{\Omega})$ of the semilinear elliptic eigenvalue problem of the form

$$
-\Delta u=\lambda f(u) \text { in } \Omega, \quad u=0 \text { on } \partial \Omega,
$$

where $\Omega$ is a bounded domain in $\mathbb{R}^{n}(n \geq 1)$ with $\partial \Omega$ smooth, $\lambda>0$, is a real bifurcation parameter, $f$ is a $C^{1}$ nonlinearity, and there are numbers $0<a_{0}<a_{1}$ such that the following conditions are satisfied:

(f1) $f(0) \geq 0$, or $\left(\mathrm{fl}^{\prime}\right) f(s)>0$ on $\left(0, a_{0}\right)$;

(f2) $f\left(a_{0}\right)=f\left(a_{1}\right)=0$;

(f3) $\max \left\{F(s): 0 \leq s \leq a_{0}\right\}<F\left(a_{1}\right)$, where $F(s) \equiv \int_{0}^{s} f(\sigma) d \sigma$.

Note that (f1) and $\left(\mathrm{fl}^{\prime}\right)$ allow $f(0)>0$ and $\left(\mathrm{fl}^{\prime}\right)$ implies (f1).

(C) 1991 Australian Mathematical Society 0263-6115/91 \$A2.00+0.00 

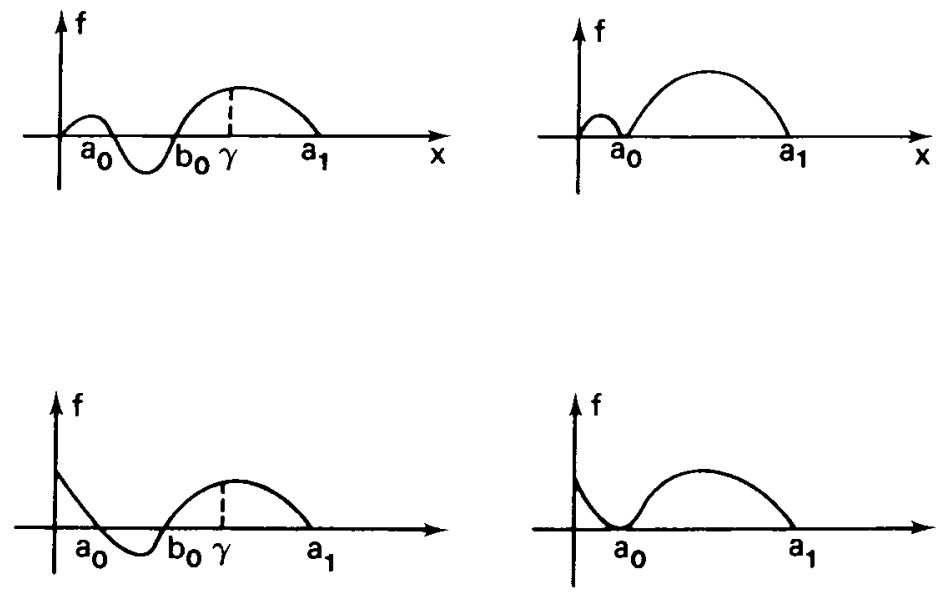

FIgURE 1. Typical $f$ 's

In part of our work we allow $f$ to change sign on $\left(a_{0}, a_{1}\right)$, and then we assume $f$ also satisfies

(f4) there exists $b_{0}$ in $\left(a_{0}, a_{1}\right), f\left(b_{0}\right)=0$, such that $\int_{a_{0}}^{b_{0}} f(s) d s<0$ and $f(s)>0$ on $\left(b_{0}, a_{1}\right)$.

If (f4) is assumed, we can find a unique $\gamma$ in $\left(b_{0}, a_{1}\right)$ such that

$$
\int_{a_{0}}^{\gamma} f(s) d s=0 .
$$

It is clear that $\lambda=0$ is not an eigenvalue of (1), and if $u$ is a positive solution of (1) satisfying $u_{\max } \in\left[a_{0}, a_{1}\right]$, then by [1, Lemma 6.2], we know $f\left(u_{\max }\right)>0$.

Four typical $f$ 's are as in Figure 1(a), (b), (c), (d).

Problem (1) has been recently discussed by E. N. Dancer [5], E. N. Dancer and K. Schmitt [6], C. Cosner and K. Schmitt [4], P. Clement and G. Sweers [3], P. Hess [8] and H. O. Peitgen, D. Saupe and K. Schmitt [10]. We note that a theorem of Smoller and Wasserman [13, Theorem 2.1] is an indirect motivation for our work.

In [5], Dancer showed that if $f$ satisfies $\left(\mathrm{fl}^{\prime}\right),(\mathrm{f} 2)$ and (f3) then for large $\lambda$, (1) has a positive solution $u(x)$ satisfying

$$
u_{\max }=\max _{x \in \Omega} u(x) \in\left(a_{0}, a_{1}\right) .
$$

In [6], Dancer and Schmitt showed that if $f$ satisfies (f2)-(f4), then the positive solution of (1) with $u_{\max } \in\left(b_{0}, a_{1}\right)$ satisfies

$$
u_{\max } \geq \gamma
$$

for general domains $\Omega$ in $\mathbb{R}^{n}(n \geq 1)$. 
In this paper we prove a nonexistence theorem which gives a $\lambda^{*}=\lambda^{*}(f, \Omega)$ $>0$ below which problem (1) does not possess any positive solutions satisfying (3); that is, we provide a lower bound for the least positive eigenvalue of (1). If $\Omega$ is a ball in $\mathbb{R}^{n}(n \geq 1)$, and $u$ is a positive solution of (1) with $u_{\max } \in\left(b_{0}, a_{1}\right)$, we improve (4) to

$$
u_{\max }>\gamma
$$

by modifying a technique used in [6]. Finally, by degree theory we prove a corollary of the nonexistence theorem which describes the dependence of the number of positive solutions of problem (1) on $\lambda$ and we prove two nonexistence theorems for $-\Delta u=M(x, u) f(u)$.

We point our that Clement and Sweers [3] have independently shown (5) by techniques different from ours and Cosner and Schmitt's [4], provided $\Omega$ satisfies a "uniform interior sphere condition." Their method, however, seems to require more regularity of $f$.

Since $\Omega$ is a bounded domain, we can find a ball $B$ with least radius $R$ such that $\Omega \subset B$. Let $c \equiv \int_{0}^{a_{1}} f^{+}(s) d s$, where $f^{+}=\max (0, f)$. Since $f$ satisfies (f2) and (f3), we define $\lambda^{*}$ as follows:

$$
\lambda^{*}= \begin{cases}\gamma^{2} / 2 c R^{2} & \text { if } f \text { satisfies (f4) } \\ & (\text { see Figure } 1(\mathrm{a}),(\mathrm{c})), \text { where } \gamma \text { is defined by (2), } \\ a_{0}^{2} / 2 c R^{2} & \text { otherwise (see Figure } 1(\mathrm{~b}),(\mathrm{d})) .\end{cases}
$$

We first prove Theorem 2 below in Section 2 in the case where $\Omega$ is a ball in $\mathbb{R}^{n} \quad(n \geq 1)$ centered at the origin by employing the famous theorem of Gidas, Ni and Nirenberg [7] on radial symmetry of positive solutions of (1) and a lower bound theorem for $u_{\max }$. Then we use a modified technique of [6] to prove (1) has no positive solutions for $\lambda \in\left[0, \lambda^{*}\right)$ for general domains $\Omega$. We prove our lower bound theorem for $u_{\max }$ at the end of Section 2 . In Section 3, we prove a corollary establishing the dependence of the number of solutions of problem (1) on $\lambda$. Finally, in Section 4 we extend our results to equations of the form $-\Delta u=M(x, u) f(u)$.

\section{Main results}

THEOREM 1 (Nonexistence of positive solutions). There exists a number $\lambda^{*}$ defined by (6) such that problem (1), with $f$ satisfying (f2) and (f3), does not possess any positive solutions satisfying (3) if $0 \leq \lambda<\lambda^{*}$. Moreover, if $f$ also satisfies (f1), then problem (1) does not possess any positive solutions satisfying (3) if $0 \leq \lambda \leq \lambda^{*}$. 
REMARK. While it is possible to give a much shorter proof of the existence of $\lambda^{*}$, the proof here gives quite a good estimate for the best $\lambda^{*}$. For example, the table below shows that our $\lambda^{*}$ is often a much better estimate than $\lambda^{* *}=\lambda_{1} / d$, where $d=\max _{x \in\left(a_{0}, a_{1}\right)} f^{\prime}(x)$ and $\lambda_{1}$ is the first eigenvalue of Laplacian $-\Delta$ subject to Dirichlet boundary condition. Our estimate tends to be better if $n$ is small and the domain is nearly circular.

We compare $\lambda^{*}$ and $\lambda^{* *}$ in the case $f(x)=-(x-1)(x-2)(x-4)$ which gives $\gamma=2.614 \ldots, c=5.750 \ldots$, and $d=20.333 \ldots$ in the following domains $\Omega \subset \mathbb{R}^{n}\left(n=2\right.$ or 3 ) (here we assume $\int_{\Omega} d x=1$ and we may remove the requirement that $\partial \Omega$ is smooth) (cf. [2]):

TABLE 1

\begin{tabular}{|llccccc|}
\hline & $\Omega\left(f_{\Omega} d x=1\right)$ & $\lambda_{1}$ & $R^{2}$ & $\lambda^{* *}$ & $\lambda^{*}$ & $\lambda^{* *}$ \\
\hline$n=2$ & Circle & 18.168 & 0.318 & 0.894 & 1.868 & 2.089 \\
& Square & 19.739 & 0.500 & 0.971 & 1.188 & 1.187 \\
& Rectangle, 3 : 2 & 21.384 & 0.542 & 1.052 & 1.096 & 1.042 \\
\hline$n=3$ & Ball & 25.646 & 0.385 & 1.261 & 1.543 & 1.223 \\
\hline
\end{tabular}

THEOREM 2 (Lower bound for $u_{\max }$ ). Suppose $f$ satisfies (f2), (f3), and (f4). Let $\gamma$ be defined by (2). Let $u$ be a positive solution of (1) with $u_{\max } \in\left(b_{0}, a_{1}\right)$. Then $u$ satisfies (5) if $\Omega$ is a ball in $\mathbb{R}^{n}(n \geq 1)$.

Remark 1. Similarly, it is easy to show that Theorem 2 holds if $\Omega$ is an annular domain in $\mathbb{R}^{n}(n \geq 1)$ and $u$ is a positive radial solution of (1).

Remark 2. Cosner and Schmitt [4] proved (4) by an identity of Rellich for $\Omega$ satisfying some symmetry conditions. Their proof can be improved to obtain (5) if $f(0) \geq 0$. However, it seems to the authors that the requirement $f(0) \geq 0$ can not be removed.

REMARK 3. Since the parameter $\lambda$ play no role in Theorem 2, we can replace $\lambda f$ by $f$ in (1) in its proof.

Proof of Theorem 1. It is easy to see that if $\lambda=0$, there is a unique trivial solution $u \equiv 0$. We first assume, in addition to (f2) and (f3), that $f$ satisfies ( $\mathrm{f} 1$ ); that is $f(0) \geq 0$. Under this assumption, we first prove the result in the special case where $\Omega$ is a ball in $\mathbb{R}^{n}(n \geq 1)$ centered at the origin.

We assume $\Omega$ is a ball $B$ in $\mathbb{R}^{n}(n \geq 1)$ with radius $R$, centered at the origin. Suppose problem (1) has a positive solution $u$ satisfying (3) for some $\lambda, 0<\lambda \leq \lambda^{*}$. It follows from the symmetry result of the Gidas, Ni and Nirenberg Theorem ([7]) that $u$ is radially symmetric and $u$ has a unique maximum at $x=0$. Hence, $u$ is a positive solution of the following 
two-point boundary value problem:

$$
\begin{aligned}
& u^{\prime \prime}(r)+\frac{n-1}{r} u^{\prime}(r)+\lambda f(u(r))=0, \quad 0<r<R, \\
& u^{\prime}(0)=u(R)=0,
\end{aligned}
$$

and

$$
u^{\prime}(r)<0 \text { for } 0<r<R .
$$

If we multiply all the terms in (7) by $u^{\prime}$ and integrate the result, we obtain

$$
\frac{1}{2}\left[u^{\prime}(r)\right]^{2}+\int_{u(0)}^{u(r)} \lambda f(s) d s=-(n-1) \int_{0}^{r} \frac{\left[u^{\prime}(s)\right]^{2}}{s} d s \leq 0 .
$$

So

$$
\frac{1}{2 \lambda}\left[u^{\prime}(r)\right]^{2}+\int_{u(0)}^{u(r)} f(s) d s \leq 0
$$

Thus,

$$
\begin{aligned}
\frac{1}{2 \lambda}\left[u^{\prime}(r)\right]^{2} & \leq \int_{u(r)}^{u(0)} f(s) d s \\
& \leq \int_{0}^{a_{1}} f^{+}(s) d s \quad\left(u(0)<a_{1}\right) \\
& =c . \quad \text { (Here, a trick is used. })
\end{aligned}
$$

Therefore,

$$
-(2 c \lambda)^{1 / 2} \leq u^{\prime}(r) \quad\left(u^{\prime}(r)<0, \text { for } 0<r<R\right) .
$$

Integrating (12), we obtain

$$
-\int_{0}^{R}(2 c \lambda)^{1 / 2} d r \leq \int_{0}^{R} u^{\prime}(r) d r
$$

consequently,

$$
-(2 c \lambda)^{1 / 2} R \leq u(R)-u(0)=-u(0) \quad(\text { since } u(R)=0) .
$$

Thus, by (3) and Theorem 2 (proved below), we have

$$
(2 c \lambda) R^{2} \geq u(0)^{2}> \begin{cases}\gamma^{2}, & \text { if } f \text { satisfies (f4) } \\ a_{0}^{2}, & \text { otherwise. }\end{cases}
$$

Hence $\lambda>\lambda^{*}$. This contradicts the assumption $0 \leq \lambda \leq \lambda^{*}$. Theorem 1 is now proved in the special case.

We now prove Theorem 1 for a general bounded domain $\Omega$ in $\mathbb{R}^{n}(n \geq 1)$ with $\partial \Omega$ smooth. Let $u$ be a positive solution of (1). The qualitative behavior of $u$ does not change if we make a translation. Thus, we can 
assume $B$ (which we consider to be the ball with least radius $R$ that covers $\Omega)$ is centered at the origin.

Suppose problem (1), with $f$ satisfying (f1), (f2), and (f3), has a positive solution $u_{0}$ satisfying (3) for some $\lambda_{0}$, with $0<\lambda_{0} \leq \lambda^{*}\left(\lambda^{*}\right.$ is defined by (6)). Consider the boundary value problem

$$
-\Delta u=\lambda_{0} f(u), \quad x \in B, \quad u=0, \quad x \in \partial B .
$$

Define $\alpha(x)$ by

$$
\alpha(x)=u_{0}(x) \quad \text { if } x \in \bar{\Omega} ; \quad \alpha(x)=0 \quad \text { if } x \in \bar{B} \backslash \Omega .
$$

Then, since $f(0) \geq 0, \alpha(x)$ is a lower solution and $\beta(x) \equiv a_{1}$ is an upper solution (see [11]) of (16). Hence, by the Method of Lower and Upper Solutions (see [11]), problem (16) has a positive solution $v$ satisfying

$$
a_{0}<v_{\max }<a_{1}
$$

for some $\lambda_{0}$, with $0<\lambda_{0} \leq \lambda^{*}$, which contradicts what we have proved above for the special case. So if $f$ satisfies (f1), (f2) and (f3), then for general domains $\Omega$, problem (1) has no positive solutions satisfying (3) if $0 \leq \lambda \leq \lambda^{*}$. Note that condition (f1) was needed to conclude that $\alpha(x)$ is a lower solution of (16).

Next we assume that $f$ does not satisfy (f1); that is, $f(0)<0$. For any $\varepsilon>0$, we replace $f$ by $\tilde{f}(\tilde{f}=\tilde{f}(s, \varepsilon))$, where $\tilde{f} \in C^{1}$ satisfies

$$
\begin{aligned}
& \tilde{f}(s, \varepsilon) \geq f(s) \quad \text { for } 0 \leq s \leq a_{0}, \\
& \tilde{f}(s, \varepsilon)=f(s) \quad \text { for } a_{0} \leq s \leq a_{1}, \\
& \tilde{f}(0, \varepsilon) \geq 0, \quad \tilde{f}\left(a_{0}, \varepsilon\right)=\tilde{f}\left(a_{1}, \varepsilon\right)=0, \quad \text { and } \\
& c+\varepsilon=\int_{0}^{a_{1}} f^{+}(s) d s+\varepsilon \geq \int_{0}^{a_{1}} \tilde{f}^{+}(s, \varepsilon) d s \geq \int_{0}^{a_{1}} f^{+}(s) d s=c .
\end{aligned}
$$

Let $d \equiv \int_{0}^{a_{1}} \tilde{f}^{+}(s, \varepsilon) d s$. Note that $d=d(\varepsilon)$ is a function of $\varepsilon$. Care must be taken in choosing $\tilde{f}$ so that (19), especially the last line of (19), holds.

Assume (1) has a positive solution $v$ satisfying (3) for some $\lambda>0$. Clearly, $v_{\max }<a_{1}$. Then for $\lambda>0$,

$$
\Delta v+\lambda \tilde{f}(v, \varepsilon) \geq \Delta v+\lambda f(v)=0 .
$$

Hence $\alpha(x) \equiv v$ is a lower solution of

$$
\Delta u+\lambda \tilde{f}(u, \varepsilon)=0, \quad x \in \Omega, \quad u=0, \quad x \in \lambda \Omega .
$$

As before, $\beta(x) \equiv a_{1}$ is an upper solution. Hence, (21) has a solution $u$ which satisfies

$$
v(x)<u(x) \leq a_{1}
$$


that is, $u$ satisfies (3) for some $\lambda>0$. But consider problem (21); since $\tilde{f}$ satisfies (f1), (f2) and (f3), by the previous result, (21) does not possess positive solutions satisfying $(3)$ if

$$
0 \leq \lambda \leq \begin{cases}\gamma^{2} / 2 d R^{2} & \text { if } \tilde{f} \text { satisfies (f4), } \\ a_{0}^{2} / 2 d R^{2} & \text { otherwise. }\end{cases}
$$

Let $\varepsilon \rightarrow 0^{+}$. By (19) we find (1) does not possess any positive solutions if $0 \leq \lambda<\lambda^{*}$. This finishes the proof of Theorem 1 .

Proof of Theorem 2. For problem (1), suppose $f$ satisfies (f2)-(f4). Let $\gamma$ be defined by (2). Suppose $\Omega$ is a ball in $\mathbf{R}^{n}(n \geq 1)$ with radius $R$ centered at the origin, and let $u$ be a positive solution of (1) with $u_{\max } \in$ $\left(b_{0}, a_{1}\right)$. Then $u$ satisfies (11). From (11), we find that

$$
0<\frac{1}{2 \lambda}\left[u^{\prime}(r)\right]^{2} \leq \int_{u(r)}^{u(0)} f(s) d s \quad\left(\text { by }(8), u^{\prime}(r)<0 \text { for } 0<r<R\right) .
$$

Now suppose

$$
u_{\max }=u(0) \leq \gamma
$$

Then by (f4),

$$
0<\int_{u(r)}^{u(0)} f(s) d s \leq \int_{u(r)}^{\gamma} f(s) d s .
$$

Choosing $r$ so that $u(r)=a_{0}$, we obtain

$$
0<\int_{a_{0}}^{\gamma} f(s) d s
$$

which contradicts (2). So if $\Omega$ is a ball centered at the origin, we obtain (5). By looking at (1) and making a translation, we can easily show (5) holds for any ball in $\mathbb{R}^{n}(n \geq 1)$. The proof of Theorem 2 is complete.

\section{A corollary}

The previous results imply the following corollary.

CoRollary 1 (Dependence of the number of positive solutions on $\lambda$ ). If $f$ satisfies $f(u) \geq 0$ for $u \in\left[0, a_{1}\right]$ in addition to $\left(\mathrm{f}^{\prime}\right)$, ( $\left.\mathrm{f} 2\right)$, and (f3), then there exists a number $\bar{\lambda}>0$ such that problem (1) has no positive solutions satisfying (3) if $\lambda<\bar{\lambda}$, at least one positive solution satisfying (3) if $\lambda=\bar{\lambda}$, and at least two positive solutions satisfying (3) if $\lambda>\bar{\lambda}$.

(Two typical $f$ 's are given in Figure 1(b), (d).) 
Consider the map $A_{\lambda}$ on $C_{0}(\overline{\mathbf{\Omega}})=\{u \in C(\overline{\mathbf{\Omega}}) \mid u=0$ on $\partial \Omega\}$ defined by $A_{\lambda}(u) \equiv(-\Delta+\tilde{t} I)^{-1}(\lambda f(u)+\tilde{t} u), \tilde{t}>0$, is such that $\lambda f^{\prime}(y)+\tilde{t}>0$ on $\left[0, a_{1}\right]$. So solutions of (1) are fixed points of $A_{\lambda}$. The operator $A_{\lambda}$ is compact; see [5] for details.

Proof of Corollary 1. It was shown in [5] that if $f$ satisfies $\left(f 1^{\prime}\right),(f 2)$ and (f3) then (1) has at least two positive solutions satisfying (3) if $\lambda$ is large. Suppose that if $\lambda=\lambda_{d}>0$, there is one positive solution $v$ satisfying (3). Then, since $f(u) \geq 0$ for $u \geq 0, u_{-} \equiv v$ is a lower solution of problem (1) and $u_{+} \equiv a_{1}$ is an upper solution of (1) for all $\lambda>\lambda_{d}$. By the Method of Lower and Upper solutions [11] again, there is at least one positive solution satisfying (3) for problem (1) for all $\lambda>\lambda_{d}$. Now simply choose $\bar{\lambda}$ to be the infimum of all $\lambda$ such that one can find one positive solution satisfying (3) for problem (1). But for $\lambda=0$, there is a unique trivial solution $u \equiv 0$ for (1). By Theorem 1 we know that $0<\bar{\lambda}$.

If $\lambda=\bar{\lambda}$, we choose a sequence $\left\{\lambda_{n}\right\}$, that $\lambda_{n}>\bar{\lambda}, \lambda_{n} \rightarrow \bar{\lambda}$. The sequence $\left\{u_{n}\right\}$ of solutions $u_{n}$ of (1) evaluated at $\lambda=\lambda_{n}$ is relatively compact in $C_{0}(\bar{\Omega})$ (since $0 \leq u_{n}<a_{1}$ in $\Omega$ ). Hence, we may assume (for a subsequence) that $u_{n} \rightarrow u$ strongly in $C_{0}(\bar{\Omega})$. Taking the limit for $A_{\lambda_{u}}\left(u_{n}\right)=u_{n}$, we find $A_{\lambda}(u)=u$. So, if $\lambda=\bar{\lambda}$, problem (1) has at least one positive solution satisfying (3).

If $\lambda>\bar{\lambda}$, we can first assume that there are finitely many positive solutions of (1) $)_{\lambda}$ satisfying (3). Let $\bar{u} \equiv a_{1}$ and $\underline{u} \equiv u_{\bar{\lambda}}$ (we choose $u_{\bar{\lambda}}$ to be the maximal positive solution of (1) $\bar{\lambda}_{\bar{\lambda}}$ satisfying (3); $u_{\bar{\lambda}}$ exists as proved above), so $\underline{u}<\bar{u}, \underline{u}$ is a lower solution which is not a solution of $(1)_{\lambda}$, and $\bar{u}$ is an upper solution, which is not a solution of $(1)_{\lambda}$. The strong maximum principle ensures that $\underline{u}<A_{\lambda}(\underline{u})$ and $A_{\lambda}(\bar{u})<\bar{u}$ [12, page 97]. Thus, by [5, Theorem 2], $A_{\lambda}$ has at least one positive solution in $(\underline{u}, \bar{u})$ isolated in $C_{0}(\bar{\Omega})$ with Leray-Schauder degree +1 , which is stable. By using Theorem 1 and the homotopy invariance property of degree theory [12, page 131] and decreasing $\lambda$, we conclude that the sum of the degrees of the solutions of problem $(1)_{\lambda}$ in

$$
D=\left\{u \in C_{0}(\bar{\Omega}) \mid u>0 \text { in } \Omega \text { and } a_{0}<u_{\max }<a_{1}\right\}
$$

is 0 . Therefore, by the excision property of degree theory [12, page 132], there is at least one solution of $(1)_{\lambda}$ in $D$ with negative Leray-Schauder degree (which can be shown to be unstable) which hence is positive in $\Omega$ and satisfies (3). So, if $\lambda>\bar{\lambda}$, Problem (1) has at least two positive solutions satisfying (3); one is stable and one is unstable. This completes the proof of Corollary 1. 

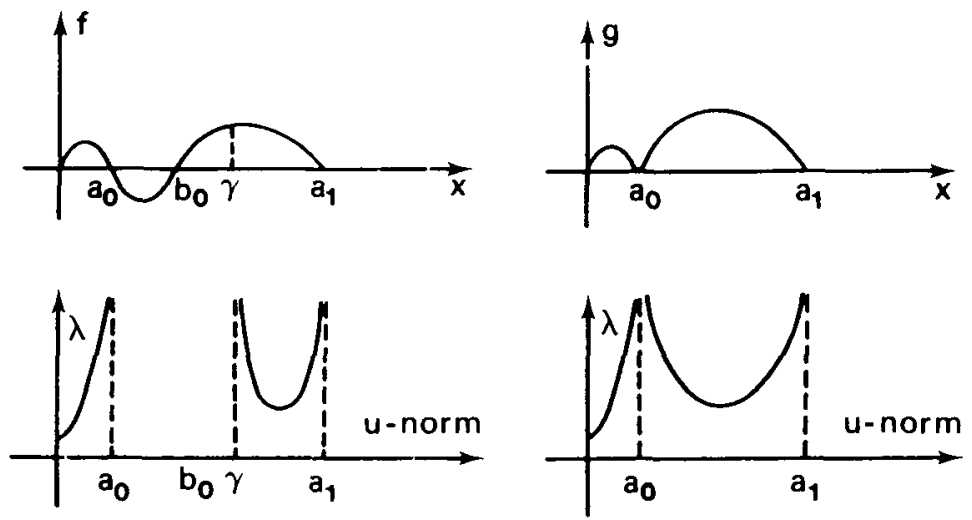

FIGURE 2. Bifurcation diagrams for the functions $f$ and $g$ shown as computed by Peitgen et al.

REMARK 1. Our results agree with the numerical results obtained by Peitgen et al. in [10], in which they chose two related nonlinearities $f$ and $g$ as in Figure 2, and used finite difference approximations to obtain numerically the set of positive solutions in some positive intervals of the corresponding boundary value problem $u^{\prime \prime}+\lambda f(u)=0, u(0)=0=u(\pi)$.

REMARK 2. For star shaped domains $\Omega, P$. L. Lions [9, Theorem 3.2] has related results without requiring $f$ to keep one sign on $\left[0, a_{1}\right]$. For general domains $\Omega$, if $f$ changes sign, the result of Corollary 1 is not known. The bifurcation diagram of problem (1) could be fairly complicated. Even though $f$ is a cubic polynomial having three distinct positive real roots and $x$ is onedimensional $(n=1)$, only some partial results are known; see [13] and [15] for references.

\section{Some extensions}

In this section we study the nonexistence of positive solutions of nonlinear elliptic eigenvalue problems of the form

$$
-\Delta u=M f(u) \text { in } \Omega, \quad u=0 \text { on } \partial \Omega,
$$

where $M=M(x, u)$ or $M(|x|, u), M>0$, and $M$ is $C^{1}$ in $u$ and $C^{\alpha}$ in $x, 0<\alpha<1$. The functions $f$ and $\Omega$ are the same as in Section 1 .

We note that, by modifying the proof of [1, Lemma 6.2], if $u$ is a positive solution of (27) satisfying $u_{\max } \in\left[a_{0}, a_{1}\right]$, we can show that $f\left(u_{\max }\right)>0$. 
Analogously to the definition of $\lambda^{*}$ in (6), we define $\lambda_{0}^{*}$ as follows:

$$
\lambda_{0}^{*}= \begin{cases}b_{0}^{2} / 2 c R^{2} & \text { if } f \text { satisfies (f4) (see Figure 1(a), (c)), } \\ a_{0}^{2} / 2 c R^{2} & \text { otherwise (see Figure } 1(\mathrm{~b}),(\mathrm{d}) \text { ). }\end{cases}
$$

We have obtained two nonexistence theorems and one existence theorem for some classes of functions $M$ and $f$ and for some domains $\Omega$. First, by modifying the proof of Theorem 1 , we are able to show our Theorem 3 , in which $M=M(|x|, u), f$ satisfies (f2) and (f3), and $\Omega$ is a bounded domain which is symmetric with respect to the origin. Finally, we also prove a nonexistence theorem and an existence theorem for (27) for general domains $\Omega$ in which, in addition to (f1), (f2), and (f3), $f$ also satisfies $f(u) \geq 0$ for $u \geq 0$.

We now consider

$$
-\Delta u=M f(u) \text { in } \Omega, \quad u=0 \text { on } \partial \Omega,
$$

where we assume $\Omega$ is symmetric with respect to the origin; that is, $x \in \Omega$ implies $-x \in \Omega$. As before we can find a ball centered at the origin with smallest radius $R$ to cover $\Omega$. We also assume that $M=M(|x|, u):(0, R) \times$ $\left(0, a_{1}\right) \rightarrow \mathbb{R}, M>0$ and satisfies

(M1) $M \in C^{1}$ in $u$ and $C^{\alpha}$ in $x, 0<\alpha<1$, and

(M2) $M$ is decreasing in $r=|x|, 0<r<R$.

Note that (M1) and (M2) are needed in applying the Gidas, Ni and Nirenberg Theorem [7]. Let $\max M(0, y)=\lambda_{0}$, for $0 \leq y \leq a_{1}$.

Modifying the proof of Theorem 1, we obtain

THEOREM 3 (Nonexistence of positive solutions). There exists a number $\lambda_{1}^{*}$ defined by (28) such that problem (29), with $f$ satisfying (f2), (f3) and $M(|x|, u)$ satisfying (M1), (M2), does not possess any positive solution satisfying (3) if $0<\lambda_{0}<\lambda_{0}^{*}$. Moreover, if $f$ also satisfies (f1), then problem (1) does not possess any positive solution satisfying (3) if $0<\lambda_{0} \leq \lambda_{0}^{*}$.

Proof of Theorem 3. The proof of Theorem 3 is similar to that of Theorem 1. Thus, we only point out the differences; these lie in obtaining results analogous to (9), (10) and (14). First, assume $f$ satisfies (f1), (f2), and (f3), and $\Omega$ is a ball centered at the origin. Suppose problem (29) has a positive solution satisfying (3). Then the Gidas, Ni and Nirenberg Theorem [7] applies. Thus, we obtain

$$
\text { (9') } \frac{1}{2}\left[u^{\prime}(r)\right]^{2}+\int_{0}^{r} M(t, u(t)) f(u(t)) u^{\prime}(t) d t=-(n-1) \int_{0}^{r} \frac{\left[u^{\prime}(s)\right]^{2}}{s} d s \leq 0 .
$$


From $\left(9^{\prime}\right)$, we know

$$
\begin{aligned}
0 & >\int_{0}^{r} M(t, u(t)) f(u(t)) u^{\prime}(t) d t \\
& \geq \int_{0}^{r} M(t, u(t)) f^{+}(u(t)) u^{\prime}(t) d t \quad\left(M>0 \text { and } u^{\prime}<0\right) \\
& =M(d, u(d)) \int_{0}^{r} f^{+}(u(t)) u^{\prime}(t) d t \text { (for some } d, 0<d<r ; \text { by } \\
& \quad \text { the Mean Value Theorem for Integrals) } \\
& \geq M(0, u(d)) \int_{0}^{r} f^{+}(u(t)) u^{\prime}(t) d t \quad\left(\text { by }(\mathrm{M} 2) \text { and } \int_{0}^{r} f^{+}(u(t)) u^{\prime}(t) d t<0\right) \\
& \geq \lambda_{0} \int_{u(0)}^{u(r)} f^{+}(s) d s .
\end{aligned}
$$

So, by the above, we obtain

$$
\frac{1}{2 \lambda_{0}}\left[u^{\prime}(r)\right]^{2}+\int_{u(0)}^{u(r)} f^{+}(s) d s \leq 0
$$

(note the difference between $(10)$ and $\left(10^{\prime}\right)$ ). Therefore,

$$
\begin{aligned}
\frac{1}{2 \lambda_{0}}\left[u^{\prime}(r)\right]^{2} & \leq \int_{u(r)}^{u(0)} f^{+}(s) d s \\
& \leq \int_{0}^{a_{1}} f^{+}(s) d s \quad\left(u(0)<a_{1}\right) \\
& =c .
\end{aligned}
$$

Following the argument between (11) and (14) of the proof of Theorem 1, we only obtain

$$
\left(2 c \lambda_{0}\right) R^{2} \geq u(0)^{2}> \begin{cases}b_{0}^{2}, & \text { if } f \text { satisfies (f4) } \\ a_{0}^{2}, & \text { otherwise }\end{cases}
$$

so $\lambda_{0}>\lambda_{0}^{*}$, which is slightly different from (15). This contradicts the assumption $0<\lambda_{0} \leq \lambda_{0}^{*}$. The case in which $\Omega$ is a ball is finished.

The rest of the proof for the cases in which $\Omega$ is not a ball and $f$ does not satisfy (f1) is quite similar to that in Theorem 1 . The proof of Theorem 3 is complete.

Applying the Method of Lower and Upper Solutions [11] again with Theorem 1 , we can easily obtain the following for general smooth bounded domains $\Omega$.

THEOREM 4 (Nonexistence of positive solutions). In addition to $\left(\mathrm{fl}^{\prime}\right)$, (f2), and (f3), if $f$ satisfies $f(u) \geq 0$ for $u \in\left[0, a_{1}\right]$, then problem (27) 
does not possess any positive solutions satisfying (3) if $\sup M(x, y) \leq \lambda^{*}\left(\lambda^{*}\right.$ is defined by (6)) for $(x, y) \in \Omega \times\left(0, a_{1}\right)$.

Similarly, applying the Method of Lower and Upper Solutions [10] again with Dancer's [5, Theorem 3], we can easily obtain the following for general smooth bounded domains.

ThEOREM 5 (Existence of positive solutions). In addition to ( $\left.\mathrm{f}^{\prime}\right)$, (f2), and (f3), if $f$ satisfies $f(u) \geq 0$ for $u \in\left[0, a_{1}\right]$, then problem (27) possesses at least one positive solution satisfying (3) if there exists a positive number $\tilde{\lambda}$ (cf. [8, page 952]), large enough, such that $\tilde{\lambda}<\inf M(x, y)$ for $(x, y) \in$ $\Omega \times\left(0, a_{1}\right)$.

\section{Acknowledgement}

The authors thank Professor E. N. Dancer for the many valuable comments and suggestions that led us easily to the proof of Corollary 1 and Professor C.-H. Lin for useful conversations on spectral theory.

\section{References}

[1] A. Ambrosetti and P. Hess, 'Positive solutions of asymptotically linear elliptic eigenvalue problems', J. Math. Anal. Appl. 73 (1980), 411-422.

[2] C. Bandle, Isoperimetric inequalities and applications, (Pitman, Boston, London, Melbourne, 1980).

[3] P. Clement and G. Sweers, 'Existence and multiplicity results for a semilinear elliptic equation', Ann. Scuola Norm. Sup. Pisa Cl. Sci. 14 (1988), 97-121.

[4] C. Cosner and K. Schmitt, 'A priori bounds for positive solutions of a semilinear elliptic equations', Proc. Amer, Math. Soc. 95 (1985), 47-50.

[5] E. N. Dancer, 'Multiple fixed points of positive mappings', J. Reine Angew. Math. 352 (1986), 46-66.

[6] E. N. Dancer and K. Schmitt, 'On positive solutions of semilinear elliptic equations', Proc. Amer. Math. Soc. 101 (1987), 445-452.

[7] B. Gidas, W.-M. Ni and L. Nirenberg, 'Symmetry and related properties via the maximum principle', Comm. Math. Phys. 68 (1979), 209-243.

[8] P. Hess, 'On multiple positive solutions of nonlinear elliptic equations', Comm. Partial Differential Equations 6 (1981), 951-961.

[9] P. L. Lions, 'On the existence of positive solutions of semilinear elliptic equations', SIAM Rev. 24 (1982), 441-467.

[10] H. O. Peitgen, D. Saupe and K. Schmitt, 'Nonlinear elliptic boundary value problems versus their finite difference approximations', J. Reine Angew. Math. 322 (1980), 75-117. 
[11] K. Schmitt, 'Boundary value problems for quasilinear second order elliptic equations', Nonlinear Anal. 2 (1978), 263-309.

[12] J. Smoller, Shock waves and reaction-diffusion equations (Springer-Verlag, New York, 1983).

[13] J. Smoller and A. Wasserman, 'Global bifurcation of steady-state solutions', J. Differential Equations 39 (1981), 269-290.

[14] G. Sweers, 'Some results for a semilinear elliptic problem with a large parameter', Proc. ICIAM 87, 1987.

[14] S.-H. Wang, 'A correction on a paper by J. Smoller and A. Wasserman', J. Differential Equations 77 (1989), 199-202.

Department of Mathematics

National Tsing Hua University

Hsinchu

Taiwan 300, R.O.C.
Department of Mathematics

State University of New York

Buffalo, New York, 14214-3093

U.S.A. 